\title{
A revolution in the simulations of super-cooled liquids and glasses
}

\author{
Models and algorithms for the next generation of glass transition studies \\ Authors: Andrea Ninarello, Ludovic Berthier, and Daniele Coslovich \\ Physical Review X 7.2 (2017): 021039. \\ Recommended with a Commentary by Giulio Biroli, École \\ Normale Supérieure, Paris, France
}

Almost any liquid when cooled fast enough to avoid the crystallisation transition enters in a metastable super-cooled regime in which the viscosity increases very rapidly. The relaxation time becomes so large in this regime, e.g. hours or days, that eventually (depending on the cooling rate and the patience of the experimentalist) super-cooled liquids fall out of equilibrium and form amorphous solids, called glasses. Understanding the physical mechanisms behind the glass transition and the nature of the glass state has motivated a vast amount of work since the late 50's [1]. There are several reasons for that. On the one hand, amorphous states of matter, in particular amorphous solids, emerge in many different contexts and across many different scales from angstrom to millimetres and from hard to soft condensed matter (e.g. molecular glasses, colloidal glasses, granular media,..). On the other hand, theoretical research on glassy systems has shown that the problem of the glass transition goes well beyond physics and finds ramifications and applications in disparate fields, such as computer science and biology. There has been a great amount of progress along the years but several questions remain unanswered. What makes the problem so difficult to study, both in experiments and simulations, is also what makes it so interesting: the relaxation time that literally blows up when temperature is decreased by a relatively small amount. Simulations of models of super-cooled liquids can "only" track the first 4-5 decades of slowing down, and are therefore far from the regime where super-cooled liquids experimentally fall out of equilibrium and become glasses. Experiments are better off, they can track 12 decades of slowing down. However, contrary to simulations, they cannot probe the microscopic motion and the complex high-order correlation functions that have been identified as relevant in the literature. Moreover, much more deeply equilibrated super-cooled liquid would be needed to obtain results as clear-cut as for usual second-order phase transitions (this is possibly due to the activated dynamics scaling associated to the glass transition). Ninarello, Berthier and Coslovich found a way to circumvent in numerical simulations this major hurdle. They have been able to achieve 10 orders of magnitude gain in the equilibration time scale, thus reaching in simulations an unprecedented degree of super-cooling, even higher (and by far) than experimental ones. 
Finding smart Monte-Carlo (MC) algorithms able to equilibrate numerically models of super-cooled liquids at low temperature is an old quest. Ninarello, Berthier and Coslovich reversed the problem, and instead of searching the best algorithm for a given system they introduced new models of super-cooled liquids for which a given algorithm provides groundbreaking results. The algorithm they focus on is the so-called swap MC where translational particle displacements are complemented by swaps of particle pairs. The method was first introduced to study simple fluids and crystals $[2,3]$ and then applied to models of super-cooled liquid in [4], for which it was shown to achieve an interesting but limited gain in equilibration time scale of the order of 2 decades. Ninarello, Berthier and Coslovich introduced new models of glass-formers: polydisperse mixtures, in which the combined optimization of the inter-particle pair-wise interaction's softness, polydispersity, and non-additivity lead to a massive gain in the equilibration time. The main idea is using continuous poly-disperse sys-

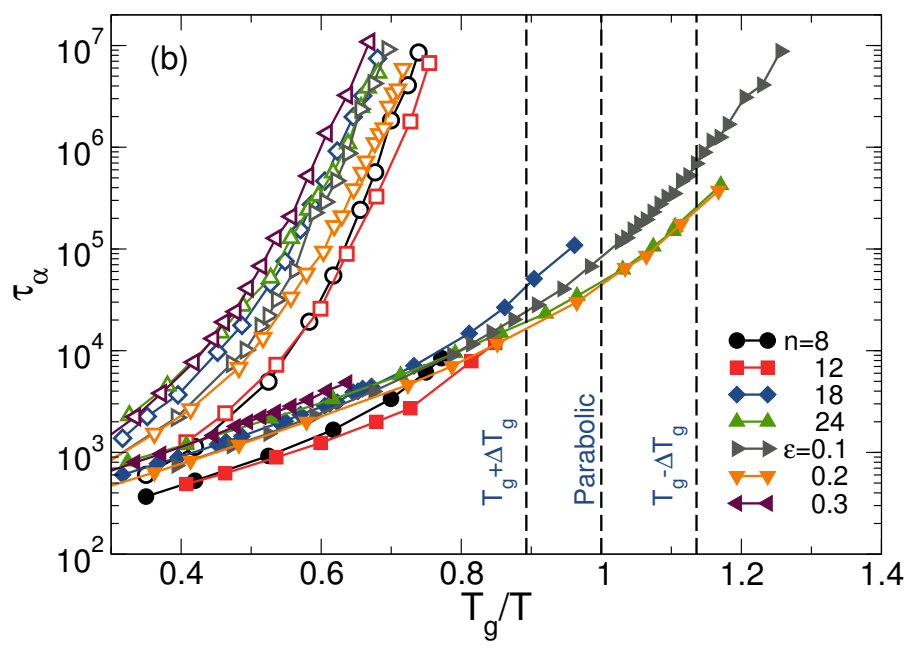

Figure 1: Relaxation times obtained from standard (open symbols) and swap (filled symbols) dynamics for various polydisperse models of various softness (n) and nonadditivity $(\varepsilon)$ are shown in an Arrhenius form with rescaled temperature $T_{g} / T$. The standard dynamics relaxation times were fitted with the Vogel-Fulcher-Tamman, parabolic $\left(\tau=\tau_{0} \exp \left(A / T+B / T^{2}\right)\right)$, and Arrhenius laws. The location of the experimental glass temperature $T_{g}$, shown with vertical dashed lines, was estimated as the temperature at which the fitted relaxation time is $10^{12}$ larger than the microscopic time (the fits are not shown in the figure).

tems, which ensures that swap MC moves are accepted frequently. In this way, particles that would be otherwise stuck for a long time in the cage formed by their neighbours are "teletransported" away. These moves makes the density field, and the system as a whole, relax much more quickly. Fig.1 (from the recommended manuscript) compares the relaxation-time obtained using a standard physical dynamics (empty symbols) with the one obtained using swap MC (filled symbols) for several models. Fig. 1 shows that thanks to the swap MC one can obtain equilibrated configurations of super-cooled liquids in a regime in which the physical dynamics would be several decades slower, making possible to explore temperatures even lower than the ones reached in experiments. The experimental glass transition temperature, $T_{g}$, is estimated in Fig.1 as the point at which the relaxation time of physical dynamics is $10^{12}$ larger than the microscopic time. 
This advance has opened the way to several investigations that were just impossible before. It is well known from experiments that amorphous solids display properties which are unlike the ones of their ordered counterparts, i.e. crystals. Thanks to the swap MC method is now possible to study such properties in simulations, and concomitantly develop theories whose microscopic predictions can be directly tested. This represents a very promising research program which is already underway with new results coming out every month, e.g. on the yielding transition [5], on the low-energy excitations [6, 7] and on the jamming transition $[8,9,10]$ of very stable glasses. Another front on which the swap MC allows to obtain crucial new information is glass-formation since it is now possible to approach much closer the putative ideal glass transition. Very interesting results have been already obtained in $[11,12]$, where it was shown that the behavior of super-cooled liquids in three dimensions, at temperatures lower than the experimental $T_{g}$, is compatible with the existence of an ideal (thermodynamic) glass transition. In two dimensions, instead, the results support the absence of a finite temperature transition, as it was expected on theoretical grounds but never confirmed until now.

As often happens, when research advances new questions arise. In particular, it is natural to ask what the impressive performance of swap MC teaches us about the nature of the glass transition, and whether there is an inherent physical difference between models for which swap MC works and models for which does not. On these and other questions, works are ongoing $[13,14]$.

\section{References}

[1] L. Berthier and G. Biroli, Rev. Mod. Phys. 83, 587 (2011).

[2] D. Gazzillo and G. Pastore, Chem. Phys. Lett. 159, 388 (1989).

[3] W.G.T. Kranendonk, D. Frenkel, Molecular physics 72 (3), 679, (1991).

[4] T. S. Grigera and G. Parisi Phys. Rev. E 63, 045102(R) (2001).

[5] M. Ozawa, L. Berthier, G. Biroli, A. Rosso, and G. Tarjus, PNAS 115 (26) 6656 (2018).

[6] L. Wang, A. Ninarello, P. Guan, L. Berthier, G. Szamel, and E. Flenner, arXiv:1804.08765

[7] G. Kapteijns, W. Ji, C Brito, M. Wyart, E. Lerner, arXiv preprint arXiv:1808.00018

[8] L. Berthier, D. Coslovich, A. Ninarello, and M. Ozawa, Phys. Rev. Lett. 116, 238002 (2016).

[9] L. Berthier, P. Charbonneau, Y. Jin, G. Parisi, B. Seoane, and F. Zamponi, PNAS 113, 8397 (2016).

[10] C. Brito, E. Lerner, M. Wyart Physical Review X 8 (3), 031050 (2018).

[11] L. Berthier, P. Charbonneau, D. Coslovich, A. Ninarello, M. Ozawa, and S. Yaida, PNAS 114, 11356 (2017). 
[12] L. Berthier, P. Charbonneau, A. Ninarello, M. Ozawa, S. Yaida, arXiv:1805.09035.

[13] M. Wyart, M.E. Cates, Physical Review Letters 119 (19), 195501 (2017)

[14] L. Berthier, G. Biroli, J.-P. Bouchaud, and G. Tarjus, arXiv:1805.12378. 